\title{
Initial Hospital Care of Spinal Cord Injury Patients
}

\author{
P. Dollfus, M.D., ${ }^{1}$ and R. Gschaedler, M.D. ${ }^{2}$ \\ ${ }^{1}$ Medical Rehabilitation Unit, Centre de Readaptation, Mulhouse, France \\ ${ }^{2}$ Traumatology Resuscitation Unit, Centre Hospitalier Louis Pasteur, Colmar, \\ France
}

The initial management of a patient with a spinal cord injury (SCI) depends on the level, and the extent of the lesion, also the site and gravity of associated lesions, and on the age and the previous medical history of the patient.

Comprehensive SCI care systems have proved their efficiency beyond doubt, but unfortunately in many countries, even in the most developed, the patient currently is still subjected to a considerable number of dangerous hazards which can not only aggravate the primary spinal cord/or nerve root lesion, resulting in secondary spinal neural damage. Delay in transportation to and from local hospitals enhance these risks by physical, medical or surgical mishandlings as lack of knowledge can cause severe complications, which can be irreversible. This fragmentation of management has been repeatedly condemned by Sir Ludwig Guttmann (1973) and others, and might impede our hopes in evaluating new SCI therapies (Pappius; 1985) (Faden, 1986).

New sophisticated diagnostic tools (CT-scan, Magnetic Resonance Imaging, Somatosensory Evoked Potentials, etc.) even when available, must not supersede the initial and repeated clinical neurological examination, which needs experience and must be performed before and after any attempt, either surgical or conservative, in obtaining reduction and fixation of the osteo-articular lesion. Spinal cord injury must never be overlooked in an unconscious patient; clinical signs of SCI often exist, even in the presence of a severe head injury. In these cases, X-ray examination of the whole spine is mandatory as an emergency procedure, as well as recognising multiple injuries (Rogers, 1984).

Treatment of the urinary bladder and sphincter impairment must be started immediately after injury. Different methods of drainage, continuous or intermittent, will be described elsewhere in this Journal. Even in the best of hands, they can usually only minimise the risk of urinary tract infection. The recent introduction of sonography not only used as a non-invasive diagnostic tool to detect internal complications, can be useful to assess the degree of early suspected urinary complications. Sterile continuous drainage systems are indis- 
pensable to control the early diuresis and fluid balance in cases of associated internal or severe peripheral lesions. The early use of prophylactic antibiotics, or chemotherapy for the urinary system is still a matter of debate.

Respiratory impairment, either neurogenic or caused by an associated chest lesion must be diagnosed and treated before it leads to acute respiratory failure. The use of respiratory physiotherapy must not be neglected and is not only performed by physiotherapists, but must be familiar to all members of the staff around the clock. If severe neurogenic respiratory insufficiency is diagnosed, the use of an appropriate assistance must not be delayed. There is still controversy between the early use of tracheotomy or naso-endotracheal intubation using low pressure high volume balloons (Gschaedler et al., 1979) (Barois et al., 1981). It depends on the appropriate SCI expertise of the team including the adequate surveillance of the acid base balance and of the blood gases. Overhydratation in those with cervical lesions must be avoided at all costs (Dietz et al., 1986).

Concerning the gastro-intestinal tract we are only beginning to understand the complexity of its neurophysiopathology, the pharmacological aspects and their inter-relationship with other physiological disturbances during the spinal shock phase. The management of gastric dilatation, frequent in those with high lesions, and often associated with paralytic ileus, must be recognised and treated as well as the management of fluid-electrolytic disturbances. Adequate nutrition is essential, and this aspect has been overlooked in the past. A minimum of 23000 calories, per 24 hours, is necessary as early as possible by parenteral (in high lesions, in the lowest possible volume) followed by enteral nutrition. This attitude is probably helpful in preventing the development of stress ulcers (Dietz et al., 1986) with the use of anti-H2 receptor drugs. The psychological team approach must never be neglected. The use of cholinergic drugs to re-establish the intestinal transit is now under debate as to their efficiency and their interaction with other pharmacological agents, especially in tetraplegic patients (mainly to increase bronchial secretions).

The prevention of bed sores and early deformities has been extensively codified by many authors in this Journal. We must not forget that the patient whose vertebral-ligamentary damage has been 'stabilised' must be handled with the same expert care as a patient treated by orthopaedic methods during the recumbency phase. The presence of multiple skeletal fractures can complicate nursing procedures. Various specialised beds and frames have been invented, to facilitate easier and safer nursing and to limit any discomfort of the patient. All have their specific advantages but some are expensive. Experience has shown the benefit of the 'log rolling technique' on an ordinary bed and an adequate mattress by a trained nursing team, even with a reduced staff. It is only recently that the evaluation of mattresses and their overlays has been studied (Krouskop et al., 1986). Seiler et al. (1986) has shown the benefit of the $30^{\circ}$ laterally inclined position when turning the patient on to his side. The frequency of alternating gentle posture of the lower limbs during each 24 hours is as important, if not more so than passive movements which are time consuming. Even more important are the posturing and early physiotherapy given to the upper limbs in tetraplegics. Painful deformities can occur rapidly, and we must not forget with the progress of modern upper limb surgery in patients with high 
spinal cord lesions that even 'a little means a lot'. We have used the possible value of $10-15^{\circ}$ body proclivity in preventing, at least partially, the effects of peripheral vasoplegia as well as an additional means of preventing deep vein thrombosis (DVT) in these very high risk patients. If physical methods, have also been used, including functional electrical stimulation, non-invasive DVT detection techniques have shown their efficiency (Chu et al., 1985). The recent introduction of new biological detection tests can be of value (for example, protein $\mathrm{C}$ and $\mathrm{S}$ ). The use of Low Molecular Weight Herparin derivatives seems promising (Henon, 1986) but needs further comparative investigations, especially in SCI patients.

\section{Conclusion}

This presentation can only be a very partial overview of some of the aspects in the initial management which has many other facets and difficulties according to 'each individual patient' (Bedbrook, 1985); and I would refer to papers published in this Journal during the past 25 years.

Unfortunately there is often a lack of appropriate teaching and proper training of the staff directly involved with these patients. This problem occurs even in the most advanced centres throughout the world, mainly due to the rapid turnover of staff. Treatment must not only be limited to a specialised group of therapists, but should be extended by the simplest and most understandable manner to those in other medical and paramedical disciplines who may possibly be involved. This can be very difficult but remains the duty of doctors specialising in the care of the spinal paralysed.

\section{References}

BEDBROOK G 1985 A balanced viewpoint in the early management of patients with spinal injuries who have neurological damage. Paraplegia 23:8-15

Chu DA, Ahn JH, Ragnarsson KT, et al. 1985 Deep venous thrombosis: diagnosis in spinal cord injured patients. Archives of Physical Medicine $\mathcal{G}$ Rehabilitation 66:365-368

DiETZ JM, BERTSCHy M, GsChAEDLER R, et al. 1986 Reflections on the intensive care of 106 acute cervical spinal cord injuries in the resuscitation unit of a general traumatology centre. Paraplegia 24:343-349

FADEN AI 1986 Neuropeptides and central nervous system injury. Archives of Neurology 43:501504

GsChaedler R, Dollfus P, Mole JP, et al. 1979 Reflections on the intensive care of acute cervical spinal cord injuries in a general traumatology centre. Paraplegia 17:58-61

Guttmann L 1973 Spinal cord injuries. Comprehensive management and research. Blackwell Scientific Publications, Oxford

HENON P 1986 Personal communication

KrousKoP TA, Williams R, KREBS M, et al. 1985 Effectiveness of mattress overlays in reducing interface pressures during recumcency. Fournal of Rehabilitation Research and Development 22:710

PAPPIUS HM 1985 The therapeutic effects of drugs in injured central nervous system. Central Nervous System 2:93-98

ROGERS LF 1984 Common oversights in the evaluation of the patient with multiple injuries. Skeletal Radiology 12:103-111

SeILER WO, Allen S, STÄHELIN HB 1986 Influence of the $30^{\circ}$ laterally inclined position and the "Super-Soft" 3-piece mattress on skin oxygen tension on areas of maximum pressure. implications for pressure sore prevention. Gerontology 32:158-166 